\title{
Observations of Systematic Errors in Calibrator Tablet Testing
}

\author{
John Burmicz \\ Pharma Test Apparatebau GmbH \\ Siemensstrasse 5, D-63512, Hainburg, Germany
}

email:drjohn.burmicz@pharma-test.de
T: he calibration of dissolution baths or rather suitability testing using calibrator tablets available from the USP, is a subject that has provoked probably the largest discussion string on various websites related to this sort of procedure. It is amazing how many people write in desperation with problems that concern suitability testing or Performance Qualification (PQ).

The purpose of this article is to provide observations in the field that may help the analyst perform calibration testing without errors.

\section{Always check the quality of the incoming tablets.}

Sometimes these are actually faulty. I have personally had tablet packages which were not only short in number but also which contained tablets in them that looked as if they had been moist at some stage with the "dried out parts" disintegrating from the main body of the tablet. When you open the package, you will find a silica gel type absorbency capsule inside. If the intended use date is some days away, some suggest that you should then place the whole container (open) with the cotton wool plug in position into a small Plexiglas dry box storage cabinet with drying agent placed below. These are conveniently available from a wide range of stockists and are really worth it for preservation of both tablets and standards. You can close the screw cap again after some hours and then replace the whole plastic bottle back in the dry box. Experience with this system is positive. In the case of Salicylic Acid tablets, be sure to carefully de-dust them prior to use. Don't use any cracked or chipped ones.

\section{Be really sure of your degassing system.}

You can use the USP method with the $0.45 \mu$ filter with preheating and vacuum. The problem is the target temperature of $41^{\circ} \mathrm{C}$. Many complaints have been received that people have to wait for the medium, once in the vessels, to fall from $39.5^{\circ} \mathrm{C}$ to (at least) $37.5^{\circ} \mathrm{C}$. In a water bath at $37.4^{\circ} \mathrm{C}$, this process may take up to 2 hours. . There is also the difference between degassing and deaeration to be considered. It was taboo at one stage to degas with helium. Degassing is the removal of dissolved gasses by substitution with another gas of lower solubility product. So nitrogen and oxygen can be displaced by helium. De-aeration is the removal of all or part of the total dissolved gasses without substitution. Today, it appears that there is a more flexible approach. As long as the deaeration method is validated, then there is generally no negative reaction.

Target oxygen levels are typically around $40-50 \%$ (ca. 3-
$4 \mathrm{mg} / \mathrm{L})$. This can be easily measured with $\mathrm{DO}_{2}$ meter. For Salicylic Acid medium, degassing is not so critical, but pH certainly is. Be sure that the bulk medium is $\mathrm{pH} 7.4 \pm 0.05$ units.

\section{Sampling.}

Be sure of the sampling system that you use. Validate, validate. Take nothing for granted. Check filter absorption. Check the clarity of the filtered sample. Some new instruments recently installed were claimed to be failing Prednisone paddle tests at $70+\%$. Apart from poor deaeration, it was remarked that the samples were cloudy even though they were filtered down to $5 \mu$. The same user also said that they did not want to put samples at an elevated temperature of ca $28^{\circ} \mathrm{C}$ directly into their $1 \mathrm{~cm}$ UV cell. So they let the solutions cool for about 30 minutes to reach room temperature. They failed. Most non-active excipients have a $D_{50}(50$ percentile particle size distribution) of 50 to $100 \mu$. The $5 \mu$ filter also has a pore size distribution, but not one which overlaps the excipient particle size to any extent. The fact that the solutions measured warm were giving results of approximately $50 \%$, but once cooled gave a result of over $70 \%$ means that active was getting through the filter. This would tend to suggest a micronized product in the mix. This effect, however, is random and can vary from bottle to bottle of Prednisone.

\section{Automation versus manual sampling.}

One could write volumes on this. Essentially, check for filter and sample tubing absorption, check and validate the pump channel flow rates, and check your flow cells. You may need either a 5 or $10 \mathrm{~mm}$ for Prednisone and a 1 or $2 \mathrm{~mm}$ cell for Salicylic Acid. Check the sample cell carrousel for its positioning in the beam. Method validation for automated measurements is essential. Do not be surprised if there is a difference between manually measured samples and their automated counterparts.

\section{Physical condition of the apparatus.}

The biggest offender here is Apparatus 1. The condition of the baskets and their clips is critical. A clear passage through any breather holes is equally critical. If you have separate spring clips and holder section in the basket assembly, make sure that the holes are aligned. In the case of misalignment, it is not unusual for Salicylic tablets to be suspended on a cushion of air and record an $11 \%$ dissolution rate (or less) instead of the nominal $23-29 \%$, if these vents are not totally clear. Align these breather holes (in the case of multi- 
component assemblies) with a simple paper clip. Also be aware of the push on types of basket as opposed to the spring clip version. Results may be lower with the push on types as the medium perturbation during the test is not so extensive. This could lead to a bi-modal distribution of results if both types are used in any one laboratory.

\section{Dissolution tester.}

When using baths with a manual (vertical) head drop, if not maintained, these may develop a rather"gritty"guide rail and thus may need a bit of pressure to pull them down into position. This could result in the basket "hitting"the medium a bit harshly and you could have a puff of disintegrating Prednisone spray out from the basket. Check out the tool shafts for bearing noise. This will not show up in a general wobble test but can arise from the passage of worn belts over the drive gears which are attached to the bearings. This "noise" (for want of a better description) can manifest itself as either a gentle "thud" or as inaudible high frequency noise, which when transmitted down the tool shaft will act almost as an ultrasonic mixer. An easy remedy for this is to spray the belts (finely) with a suitable light oil and PTFE mixture that is normally used to reduce squeal in car cam belts. Worn bearings should be changed.

\section{Vessels.}

Normally a poor vessel is clearly visible, and if scratched (especially plastic ones) should be thrown away. The centricity should be checked as this can really have a negative effect. Check that any new vessels are spherical in the base. If flat or slightly raised this could cause the tablet to race around and the dissolution rate may be greatly increased.

\section{Vibration.}

There is no specification for this yet, although a noisy pumping system, whether on board or external will certainly add to the failure possibility. The proximity of noisy equipment in the vicinity of the tester will also make a contribution. Old fridges and surface area measurement instruments (BET) with cranky old vacuum pumps, being some of the least recognized offenders. Systems that have built in vibration absorption mechanisms may be helpful or which have good quality circulators that can be bench mounted and do not transmit noise.

\section{General tips:}

Always validate the manual or automated sampling systems that you intend to use. If there is a peristaltic pump, make sure that the tubing is round and not flattened by continuous roller pressure. Validate the flow rate for each channel. The pump supplier can usually give you an opera- tion qualification (OQ) for this purpose.

Always check the tools out carefully (should be part of the $\mathrm{OQ}$ ), especially for wobble. Check baskets for the correct mesh size and integrity. Blocked mesh, corrosion, bent sides and un-welded seams are all typical faults. Check the clips for venting and equal grip from all three springs. Every degree out of kilter will add to the percentage dissolved.

Check your vessels for quality and the clearance of the tools from the vessels base.

It may be useful to know your deaeration level. Get a decent deaeration system that can provide a good quantity of medium and a quality of deaeration that can be measured. Dissolved oxygen is a method of measuring the deaeration level. You can validate your deaeration method with recorded results. After medium delivery, always cover the vessel before the test starts.

Some of these items may sound like common sense, but some of the key points are easily overlooked. This short article should at least help to formulate some of the anecdotal tales and hearsay into a validated SOP for these Calibrator Tests.



A new magazine dedicated to solid-dosage forms:

- Pharmaceuticals

- Nutraceuticals

- Chemicals

- And more!

- Vitamins

Do you qualify for a FREE subscription?

Find out at www.tabletscapsules.com

Glick on "Subscribe"

You can also e-mail us at: tc@cscpub.com

Or call +1 6512875600 for more information. 